\title{
ON HOM-LEIBNIZ AND HOM-LIE-YAMAGUTI SUPERALGEBRAS
}

\author{
Sylvain Attan $^{1}$, Donatien Gaparayi $^{2}$ \\ AND \\ A. Nourou ISSA ${ }^{1}$ \\ ${ }^{1}$ Département de Mathématiques \\ Université d'Abomey-calavi \\ 01 BP 4521, Cotonou 01, Bénin \\ ${ }^{2}$ Ecole Normale Supérieure (E.N.S), \\ BP 6983 Bujumbura, Burundi \\ e-mail: syltane2010@yahoo.fr \\ gapadona@yahoo.fr \\ woraniss@yahoo.fr
}

\begin{abstract}
In this paper some characterizations of Hom-Leibniz superalgebras are given and some of their basic properties are found. These properties can be seen as a generalization of corresponding well-known properties of HomLeibniz algebras. Considering the Hom-Akivis superalgebra associated to a given Hom-Leibniz superalgebra, it is observed that the Hom-super Akivis identity leads to an additional property of Hom-Leibniz superalgebras, which in turn gives a necessary and sufficient condition for Hom-super Lie admissibility of Hom-Leibniz superalgebras. We also show that every (left) HomLeibniz superalgebra has a natural super Hom-Lie-Yamaguti structure.
\end{abstract}

Keywords: Hom-Leibniz superalgebras, Hom-Akivis superalgebras, HomLie-Yamaguti superalgebras.

2010 Mathematics Subject Classification: 17A30, 17A32, 17D99.

\section{REFERENCES}

[1] K. Abdaoui, F. Ammar and A. Makhlouf, Hom-alternative, Hom-Malcev and HomJordan superalgebras, Bull. Malays. Math. Sci. Soc. 40 (2017) 439-472. https://doi.org/10.1007/s40840-016-0323-5 
[2] H. Albuquerque A. P. Santana Akivis superalgebras and speciality, Contemporary Math. 483 (2009) 13-22.

[3] F. Ammar and A. Makhlouf, Hom-Lie superalgebras and Hom-Lie admissible superalgebras, J. Algebra 324 (2010) 1513-1528.

https://doi.org/10.1016/j.jalgebra.2010.06.014

[4] H. Ataguema, A. Makhlouf and S.D. Silvestrov, Generalization of n-ary Nambu algebras and beyond, J. Math. Phys. 50 (2009) 083501. https://doi.org/10.1063/1.3167801

[5] D. Gaparayi and A.N. Issa, Hom-Akivis superalgebras, J. Alg. Comput. Appl. 6 (2017) $36-51$.

[6] D. Gaparayi and A.N. Issa, A Twisted Generalization of Lie-Yamaguti Algebras, Int. J. Algebra 7 (2012) 339-352.

[7] D. Gaparayi and A.N. Issa, Hom-Lie-Yamaguti structures on Hom-Leibniz algebras, Extracta Math. 28 (2013) 1-12.

[8] D. Gaparayi, S. Attan and A.N. Issa, Hom-Lie-Yamaguti superalgebras, Korean J. Math. 27 (2019) 175-192. https://doi.org/10.11568/kjm.2019.27.1.175

[9] Y. Fregier and A. Gohr, Unital algebras of Hom-associative type and surjective or injective twistings, J. Gen. Lie Theo. and Appl. 3 (2009) 285-295.

[10] J.T Hartwig, D. Larsson and S.D. Silvestrov, Deformations of Lie algebras using $\sigma$-derivations, J. Algebra 295 (2006) 314-361.

https://doi.org/10.1016/j.jalgebra.2005.07.036

[11] A.N. Issa, Some characterizations of Hom-Leibniz algebras, International Int. Elect. J. Alg. 14 (2013) 1-9.

[12] A.N. Issa, Hom-Akivis algebras, Comment. Math. Univ. Carolin. 52 (2011). http://dml.cz/dmlcz/142800.

[13] Q. Jin and X. Li, Hom-Lie algebra structures on semi-simple Lie algebras, J. Algebra 319 (2008) 1398-1408. https://doi.org/10.1016/j.jalgebra.2007.12.005

[14] M.K. Kinyon and A. Weinstein, Leibniz algebras, Courant algebroids and multiplications on reductive homogeneous spaces, Amer. J. Math. 123 (2001) 525-550. https://doi.org/10.1353/ajm.2001.0017

[15] J-L. Loday. Une version non commutative des algèbres de Lie: les algèbres de Leibniz, Enseign. Math. 39 (1993) 269-293.

[16] A. Makhlouf, Hom-alternative algebras and Hom-Jordan algebras, Int. Elect. J. Alg. 8 (2010) 177-190.

[17] A. Makhlouf, Paradigm of nonassociative Hom-algebras and Hom-superalgebras, in: Proceedings of Jordan structures in algebra and Analysis Meeting, 143-177, Editional Circulo Rojo (Almeria, 2010). 
[18] Qi Nie and Zhen Li, The Classification of 4-dimensional Leibniz Superalgebras, Math. Aeterna 72017 375-380.

[19] I.P. Shestakov, Prime Mal'tsev superalgebras, Mat. Sb 182 (1991) 1357-1366

[20] B. Sun, The construction of Hom-Novikov superalgebras, Math. Aeterna 6 (2016) 605-609.

[21] C. Wang, Q. Zhang and Z. Wei, Hom-Leibniz superalgebras and Hom-Leibniz Poisson superalgebras, Hacet. J. Math. Stat. 44 (2015) 1163-1179.

[22] D. Yau, Hom-Malcev, Hom-alternative, and Hom-Jardan algebras, Int. Elect. J. Alg. 11 (2012) 177-217.

[23] D. Yau, Hom-algebras and Homology, J. Lie Theory 19 (2009) 409-421.

[24] D. Yau, Hom-Novikov algebras, J. Phys. A 44 (2011) 085202.

[25] D. Yau, On n-ary Hom-Nambu and Hom-Nambu-Lie algebras, J. Geom. Phys. 62 (2012) 506-522. https://doi.org/10.1016/j.geomphys.2011.11.006

[26] J.X. Yuan, L.P. Sun and W.D. Liu, Multiplicative Hom-Lie superalgebra structures on infinite dimensional simple Lie superalgebras of vector fields, J. Geom. Phys. $\mathbf{8 4}$ (2014) 1-7.

https://doi.org/10.1016/j.geomphys.2014.06.001

Received 19 May 2020

Revised 3 August 2020

Accepted 3 August 2020 